\title{
An examination of school reopening strategies during the SARS-CoV-2 pandemic
}

Alfonso Landeros ${ }^{1}$, Xiang Ji ${ }^{6}$, Kenneth Lange ${ }^{1,2,5}$, Timothy C. Stutz ${ }^{1}$, Jason Xu ${ }^{7}$, Mary E. Sehl ${ }^{1,4,}{ }^{*}$, Janet S. Sinsheimer ${ }^{1,2,3,{ }^{*}}$

1 Department of Computational Medicine, UCLA, Los Angeles, CA, USA

2 Department of Human Genetics, UCLA, Los Angeles, CA, USA

3 Department of Biostatistics, UCLA, Los Angeles, CA, USA

4 Division of Hematology-Oncology, Department of Medicine, UCLA, Los Angeles, CA, USA

5 Department of Statistics, UCLA, Los Angeles, CA, USA

6 Department of Mathematics, Tulane University, New Orleans, LA, USA

7 Department of Statistical Science, Durham, Duke University, NC, USA

* To whom correspondence should be addressed; msehl@mednet.ucla.edu, jsinshei@ucla.edu

5357 Gonda Building

695 Charles E. Young Drive South

Los Angeles, CA 90095-1766

$+1(310)$ 825-8002

\section{Abstract}

The SARS-CoV-2 pandemic led to closure of nearly all K-12 schools in the United States of America in March 2020. Although reopening K-12 schools for in-person schooling is desirable for many reasons, officials understand that risk reduction strategies and detection of cases are imperative in creating a safe return to school. Furthermore, consequences of reclosing recently opened schools are substantial and 
impact teachers, parents, and ultimately educational experiences in children. To address competing interests in meeting educational needs with public safety, we compare the impact of physical separation through school cohorts on SARS-CoV-2 infections against policies acting at the level of individual contacts within classrooms. Using an age-stratified Susceptible-Exposed-Infected-Removed model, we explore influences of reduced class density, transmission mitigation, and viral detection on cumulative prevalence. We consider several scenarios over a 6-month period including (1) multiple rotating cohorts in which students cycle through in-person instruction on a weekly basis, (2) parallel cohorts with in-person and remote learning tracks, (3) the impact of a hypothetical testing program with ideal and imperfect detection, and (4) varying levels of aggregate transmission reduction. Our mathematical model predicts that reducing the number of contacts through cohorts produces a larger effect than diminishing transmission rates per contact. Specifically, the latter approach requires dramatic reduction in transmission rates in order to achieve a comparable effect in minimizing infections over time. Further, our model indicates that surveillance programs using less sensitive tests may be adequate in monitoring infections within a school community by both keeping infections low and allowing for a longer period of instruction. Lastly, we underscore the importance of factoring infection prevalence in deciding when a local outbreak of infection is serious enough to require reverting to remote learning.

\section{Introduction}

Reopening K-12 schools is a topic of intense discussion. Because transmission of SARS-CoV-2 occurs through respiratory droplets, reopening policies must adequately reduce crowded environments at school to protect children, teachers, staff, and ultimately communities. Unfortunately, many factors work to the detriment of ostensibly reasonable strategies, including extended hours for teachers, challenges in transporting children to and from school, and reduced quality of educational experience. Although U.S. school closures in March 2020 reduced COVID-19 cases in states with low cumulative incidence, education researchers worry about lagging educational development of children once schools reopen [1] 3 . A predictable, regular attendance policy is crucial in balancing social burden with maintaining steady educational 
progress.

As school systems, professional organizations, and governments have proposed different reopening strategies to reduce infection risks to students, teachers, school staff, and faculty, it is helpful to quantify ramifications of different plans [4]. Here we explore a simple, interpretable mathematical model that compares infection rates under various reopening scenarios. We compare consequences of (1) reopening at full capacity, (2) allowing half of all children to return to in-person schooling while the other half continues with remote learning (parallel cohorts), and (3) alternating sessions in which different student cohorts attend school every other or every third week (rotating cohorts). Our goal is to provide insight into epidemiological consequences of reopening strategies and to quantify their consequences. In particular, we explore implications of the reclosing guidelines announced by Governor Gavin Newsom for California schools [5].

\section{Methods}

\section{Compartmental Model}

Our approach uses a deterministic Susceptible-Exposed-Infected-Removed (SEIR) model stratified by age group and cohort. We assume that infecteds may or may not present with symptoms and that the removed pool accounts for individuals with negligible contribution to infection spread, including individuals that have either recovered with full immunity or died. Given that natural immunity may persist over several months 64 and that our simulations span a period of 6 months, we make the plausible assumption that individuals do not return to the susceptible pool once infected. For simplicity, the simulation scope is limited to two age groups, children in K-12 education spread over 1 to 3 child cohorts and adults over 18 years. Births are ignored because our simulations operate on relatively short time scales. Although mortality certainly represent an important metric for public health concerns, we do not model deaths explicitly. This simplification avoids introducing additional model parameters. In theory, one might approximate deaths by adjusting our predictions for the number of removed individuals by community-specific estimates for death rates. Model assumptions are further elaborated in our discussion of transmission rates and other model parameters. 
In our differential equations model the functions $S(t), E(t), I(t)$, and $R(t)$ denote the fraction of susceptible, exposed, infected, and removed individuals, respectively, in an overall population at time $t$. Each compartment is stratified by age class ( 1 for children, 2 for adults) and cohort membership so that $I_{1 k}$ refers to infected children in cohort $k$. With this notation in mind, we propose the following model for the force of infection acting on susceptible individuals in class $j$ and cohort $k$, denoted $\lambda_{j k}(t)$, as

$$
\begin{aligned}
\lambda_{j k}(t) & =\sum_{\text {cohort } \ell} \sum_{\text {age class } i}\left(\begin{array}{c}
\text { interaction } \\
\text { between } \\
\text { cohorts } k, \ell
\end{array}\right) \times\left(\begin{array}{c}
\text { transmission } \\
\text { from age } \\
\text { group } i \rightarrow j
\end{array}\right) \times\left(\begin{array}{c}
\text { fraction of } \\
\text { infecteds in } \\
\text { age group } i, \\
\text { and cohort } \ell
\end{array}\right) \\
& =\sum_{\ell} \sum_{i} \alpha_{k \ell} \times \beta_{i j} \times I_{i \ell}(t) .
\end{aligned}
$$

For pairs of cohorts $k \neq \ell$, the extremes $\alpha_{k \ell}=0$ and $\alpha_{k \ell}=1$ reflect complete separation $\quad{ }_{47}$ and mixing between two cohorts, respectively. Values in between these limits may be $\quad{ }_{48}^{48}$ interpreted as decreased interaction due to physical or social distancing. Weak cohort ${ }_{49}^{49}$ interactions are fixed at $\alpha_{k \ell}=0.05$ in all of our simulations. The transmission rates $\beta_{i j} \quad{ }_{50}$ may be asymmetric to capture heterogeneity in transmission due to different contact 51 patterns, susceptibility, or infectiousness. Lastly, the parameters $\sigma_{j}$ and $\gamma_{j}$ for age class $\quad{ }_{52}$ $j$ represent rates at which exposed individuals become infectious (latency) and infecteds $\quad{ }_{53}$ recover from the contagious stage (infectiousness), respectively. Specifically, we take $\quad 54$ $1 / \gamma_{j}$ as the average number of days an individual in class $j$ is contagious based on a $\quad 55$ time-homogeneous Markovian model; an analogous interpretation holds for the latency parameters.

Fig. 1 summarizes the high level features of our mathematical model. All numerical simulations are carried out in the Julia programming language using tools from the SciML ecosystem $13-16$.

We note that our modelling studies differ from previous work. Compared to Zhang et al. 12], our model lacks the detailed data on contact patterns among multiple age classes. This omission is deliberate. Our model focuses on the interaction between adult and child age classes to understand the influence of transmission rates, cohort structure, and demographics simultaneously. The later section on our phenomenological transmission model elaborates on this approach. Similarly, we depart from the 
framework of Lee et al. 49 to model multiple rotating cohorts and the influence of increased child-child contact due to in-person school attendance.

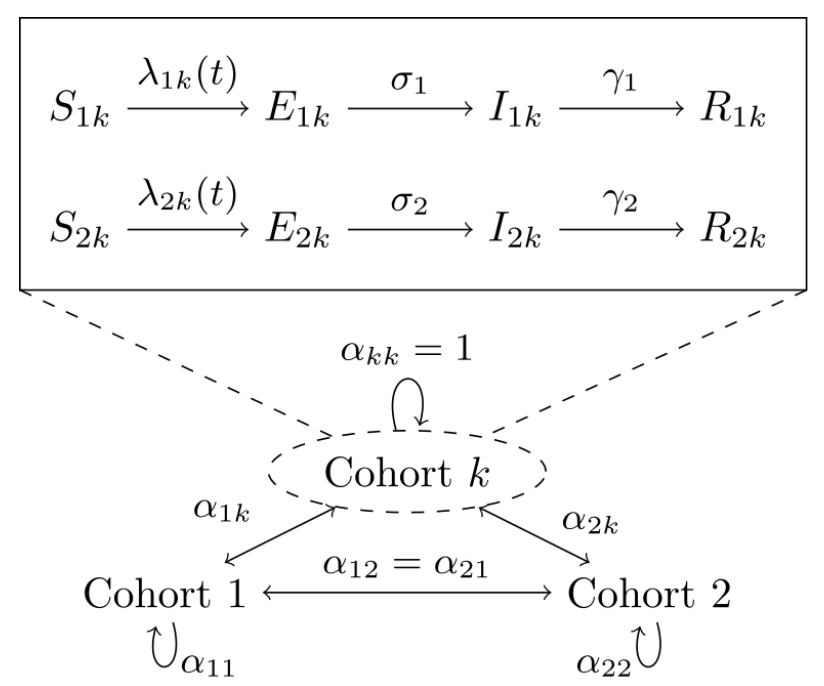

Fig 1. Overview of SEIR compartmental model. The main compartments are denoted by $S(t), E(t), I(t)$, and $R(t)$ for susceptible, exposed, infected, and removed, respectively. Compartments are stratified by age class $(1$ - children, 2 - adults) and membership to cohort $k$. The coefficients $\alpha_{k \ell} \in[0,1]$ account for the strength of interaction between cohorts $k$ and $\ell$.

\section{Simulation studies on prevalence thresholds}

We consider the effect of a stopping rule on cumulative prevalence. Inspired by California's guidelines urging schools to revert to remote learning whenever the infections within a school reach $5 \%$ in 2-week period (3), we define the stopping time $t_{\text {threshold }}$ as the first time that detected school cases reach the specified threshold.

Formally, the stopping time is given by

$$
t_{\text {threshold }}=\inf \left\{t \in T: \sum_{s \in \text { window }(t)} C(s) \geq 5 \%\right\} \text {, }
$$

where $T$ is a set of testing times and the sum is taken over a sliding 14-day window up to time $t$. The quantity $C(s)=$ sensitivity $\times I_{1 k}(s) / q$ represents detected cases adjusted for population size $q$, and is specific to the active cohort $k$. Detection necessarily depends on a particular test's sensitivity and is based on testing at the beginning of a 
and placed in the removed state $(I(t) \rightarrow R(t))$. The isolation rule applies only to the cohort at school, while the sensitivity factor in the rule captures imprecision in testing and reporting.

Note that $t_{\text {threshold }}=\infty$ if the threshold is never reached over the time span of our simulations. Furthermore, our simulation results involving $t_{\text {threshold }}$ represent a lower bound because case isolation is taken to be instantaneous. In practice, segregation of affected pupils is delayed. Our model does not explicitly account for adult staff at school. Our simplifying assumption is justified by our focus on qualitative behavior and the fact that students typically outnumber teachers and ancillary staff. For example, the average class sizes for public elementary and public secondary schools are estimated to be 21.2 and 26.8 students, respectively, for the 2011-2012 academic year 50. At a 20:1 student to staff ratio, a school with 1000 students would need 53 cases in a 14-day period to meet the closure criterion of 50 set in our simulations.

\section{Modeling transmission between age classes}

In spite of less severe disease and lower case-fatality rates than adults, children may be just as prone to SARS-CoV-2 infections as adults 20]. Children's symptoms range from fever, rhinitis, cough, and GI symptoms, to a Kawasaki-like disease termed Multisystem Inflammatory Syndrome in Children (MIS-C) 21, 39, 40. However, because children's symptoms are typically less severe and of shorter duration than those of adults [42, the likelihood of pediatric infection escaping symptom-based monitoring, such as temperature screening, is higher than that of adults. This reality increases pre-symptomatic and asymptomatic transmission 11,22. Thus, detecting transmission in children is difficult; quantifying it is all the more challenging 26.28 .

Contact tracing data from Singapore suggest that per contact transmission between children, particularly in educational settings, is low compared with adult-adult transmission [24. Yet the number of contacts between children is expected to be significantly higher compared to other age groups 12,25 . Changes in contact structure will necessarily change estimates of transmission rates. For example, Li et al. provide transmission rate estimates for Wuhan prior to (1.12 per day) and following travel restrictions (0.52 per day) 28]. An additional source of heterogeneity in transmission is 
the potentially reduced susceptibility of children compared to adults 10 . The review by ${ }^{111}$ Viner et al. summarizes much of the early literature on this topic and suggests that the ${ }_{112}$ susceptibility of adolescents may be similar to that of adults 43 . Infectiousness of ${ }_{113}$ different age groups is not as well characterized. Each source of heterogeneity poses a ${ }^{114}$ challenge to developing a parsimonious mathematical model.

Rather than reconciling transmission rate estimates across populations based on different scientific models, we vary transmission rates between and within age classes to underscore the influence of modelling assumptions on epidemiological consequences and to calibrate the range of effects given existing evidence. To this end, our transmission rates $\beta_{i j}$ are designed to separate the magnitude of transmission from the effects of different age class interactions. Scale is determined by baseline transmission rate, $\beta_{0}$ and is interpreted as a characteristic of a population. The baseline transmission rate is then used to define each $\beta_{i j}$ based on the formula $\beta_{i j}=\beta_{0} \times f_{i j}$ with weights $f_{i j} \in[0,1]$ capturing the contribution of each $i \rightarrow j$ interaction to the aggregate transmission rate $\beta_{0}$. For our model with only 2 age classes, imposing the constraint

$$
f_{11}+f_{12}+f_{21}+f_{22}=1
$$

allows us to explore the effect of transmissibility assumptions at a fixed scale while retaining the complexity of contact matrices, susceptibility, and infectiousness.

There are a few special cases to point out. The case

$$
f_{11}=f_{12}=f_{21}=f_{22}=1 / 4
$$

assumes that child-child, child-adult, adult-child, and adult-adult interactions are indistinguishable and therefore that the two age groups are equivalent on the basis of transmission. In the absence of cohort structures, the force of infection on class $j$ becomes

$$
\lambda_{j}=\beta_{0} \sum_{i} f_{i j} I_{i}=\beta_{0} I
$$

effectively collapsing our model to the basic SEIR equations under which $\beta_{0}$ is the transmission rate of a homogeneous population. The case $f_{11}<f_{22}$ reflects lower susceptibility in children compared to adults, differences in contact structure, or both. 


\section{Cohort structure and increased child-child contact}

A well-timed cyclic attendance strategy tuned to the latent period of SARS-CoV-2 may

\section{Choices for other model parameters}

In contrast to factors contributing to transmission rates, latent, infectious, and 
Other studies report serial intervals and incubation periods consistent with these estimates for latency and infectiousness [31,32]. Unfortunately, the literature on similar epidemiological inferences in children is sparse. One observational study suggests children may have incubation periods similar to those of adults 33 .

Because our simulations model school reopening, the proportion of infected individuals will influence prevalence and especially time to school closures. A periodic joint report from the American Academy of Pediatrics and Children's Hospital Association indicates that children account for 12.9\% (range: 8\%-20\%) of COVID-19 cases across US states and territories as of February 4, 2021 [44]. We also account for demographic structure by considering the proportion of children and adults in simulations. The American Community Survey Education Tabulation for 2014-2018 50] suggests that children under 18 years of age make up approximately $22 \%$ of a population delineated by school district boundaries. Thus, we calibrate our simulations to a population mix of $22 \%$ children ad $78 \%$ adults and assume children account for $10 \%$ of infections at the beginning of our simulations. In addition, the total proportion of infections is fixed to $2 \%$ of a population (2000 active infections per 100,000$)$ to simulate under conditions away from disease-free equilibrium and to ensure the stopping time is not immediately hit.

Table 1 summarizes our choices and lists references pertinent to each choice, where applicable.

\section{Results}

\section{Cohorts reduce $\mathcal{R}_{0}$ under various transmission modalities}

We first examine the impact of separating children into rotating cohorts on the basic reproduction number $\mathcal{R}_{0}$ of the model. Unfortunately, this quantity necessarily depends on poorly characterized transmission rates and varies with different contact patterns and human behaviors. Thus, we use our parameterization $\beta_{i j}=\beta_{0} f_{i j}$ to identify dominant terms $f_{i j}$ contributing to $\mathcal{R}_{0}$ under varying cohort numbers but with $\beta_{0}$ fixed. In particular, we consider 3 interesting cases: (1) adult-child and child-adult transmission are symmetric, (2) child-child transmission is weak, and (3) adult-adult transmission is 
Table 1. Summary of model parameters with ranges and estimates.

\begin{tabular}{|c|l|l|}
\hline Parameter & Description & Range/Estimate \\
\hline$\beta_{0}$ & Bulk transmission rate for population & 1.2 day $^{-1}, 1.5$ day $^{-1}$ \\
\hline$f_{11}$ & Weight for child to child transmission. & $0-1 ; 0.1$ \\
\hline$f_{12}$ & Weight for child to adult transmission. & $0-1 ; 0.25$ \\
\hline$f_{21}$ & Weight for adult to child transmission. & $0-1 ; 0.15$ \\
\hline$f_{22}$ & Weight for adult to adult transmission. & $0-1 ; 0.5$ \\
\hline $1 / \sigma_{1}$ & Average child latency period. & 3 days \\
\hline $1 / \sigma_{2}$ & Average adult latency period. & 3 days \\
\hline $1 / \gamma_{1}$ & Average child infectious period. & 4 days \\
\hline $1 / \gamma_{2}$ & Average adult infectious period. & 4 days \\
\hline$\alpha_{k k}$ & Strength of interactions within a cohort $k$. & 1 \\
\hline$\alpha_{k \ell}$ & Strength of interactions between cohorts $k$ and $\ell$. & 0.05 \\
\hline$I(0)$ & Proportion of infecteds at reopening (incidence). & $0.0-10 \% ; 2 \%$ \\
\hline$I_{1 k}$ & Proportion of infected children at reopening. & $0.0-10 \% ; 10 \%$ \\
\hline$c$ & Multiplier modeling increased child-child contact. & $1,2,10$ \\
\hline
\end{tabular}

The range for transmission between adults suggested by Li et al. $[28]$ calibrates the bulk rate. Latency and infectious period estimates are based on $\mathrm{Li}$ et al. and the summary by Bar-On et al. 28,30 . The initial proportion of infected individuals is equally distributed across cohorts.

weak. Fig. 2 summarizes the results of our analysis. In each of these cases, we find that splitting a school community into 2 or 3 rotating cohorts substantially reduces $R_{0}$ under a wide range of parameter values. For example, in the regime with symmetric between-class transmission and weak child-child transmission, moving from full capacity to 2 cohorts shifts $\mathcal{R}_{0}$ from about 3 to about 1.5 (Fig. $2 \mathrm{~A}-\mathrm{B}$, right corners). Moving further to 3 cohorts brings the reproduction number below 1 in the same regime (Fig. 2 2 ). Relaxing the symmetry assumption, we find the pattern recapitulated under both assumptions of weak child-child transmission (Fig. 2D-F) and weak adult-adult transmission (Fig. 2 $\mathrm{G}-\mathrm{I}$ ). Further, this analysis suggests that child-adult and adult-adult transmission can play dominant roles in the short-term dynamics of our model under the plausible scenario of weak child-child transmission (Fig. 2D, right and top corners). The influence of $\beta_{22}$ is not surprising because our virtual population's demography is skewed toward adults $(78 \%)$. However, our results demonstrate that child-adult transmission should be weighed carefully in reopening decisions because it less characterized and poses a potent risk, especially to school teachers.

We focus our attention on the asymmetric case with weak child-child transmission for the remainder of the study. Specifically, we set $f_{11}=0.1, f_{12}=0.25, f_{21}=0.15$, and $f_{22}=0.5$ to model this scenario, and take $\beta_{0}=1.2$ to simulate under $R_{0} \approx 3$. This 

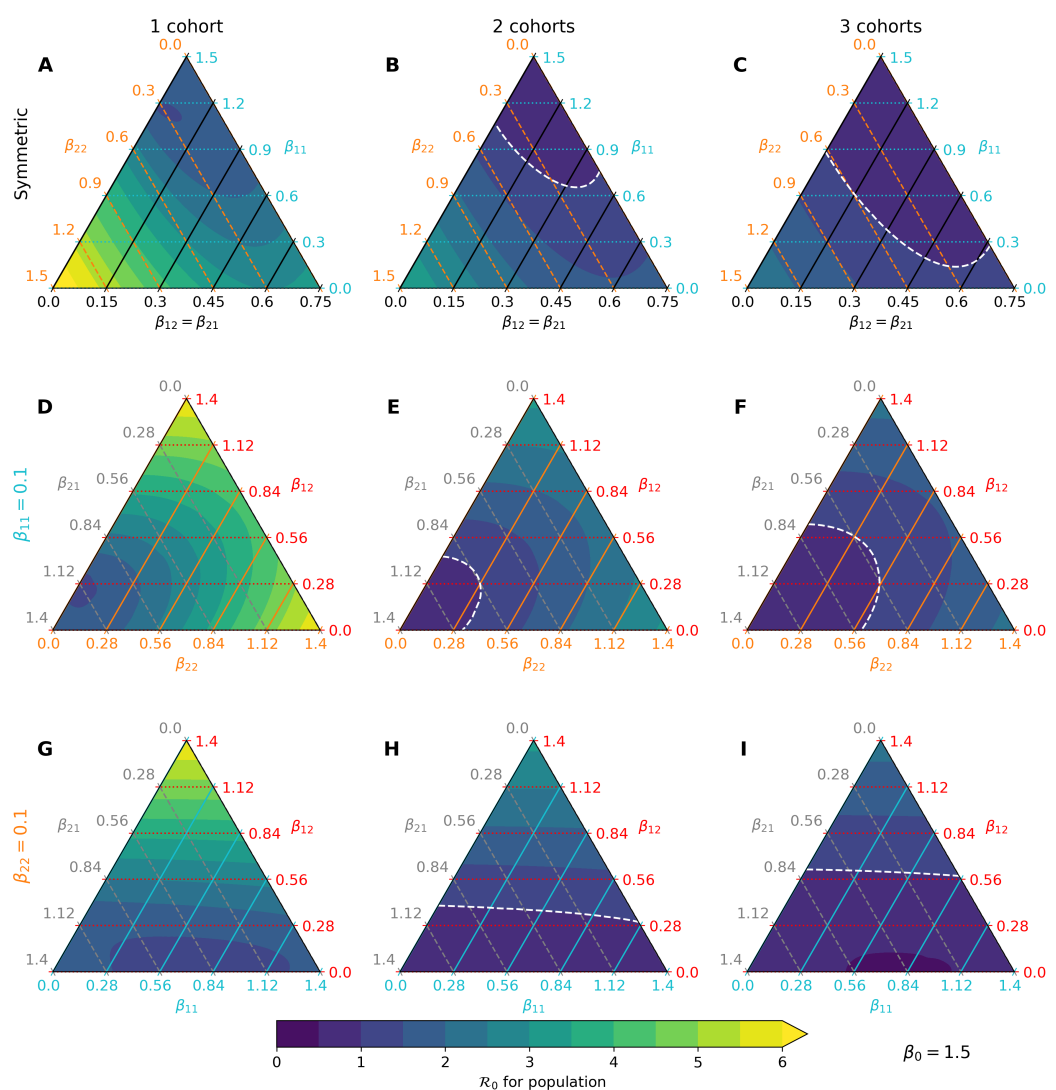

Fig 2. Predicted $\mathcal{R}_{0}$ under various transmission-cohort scenarios. The color gradient changes from purple to blue to reflect $R_{0}$ shifting from $<1$ to $>1$ in each ternary plot, with the white line denoting the boundary. Yellow is used to represent $R_{0}>6$. (A-C) Assuming child-adult and adult-child transmission rates are identical (black axis), movement along the blue axis indicates that child-child transmission has a weak effect on $R_{0}$ at a fixed scale for $\beta_{0}$. (D-F) Fixing child-child transmission to be weak $\left(\beta_{11}=0.1\right)$ relative to other interactions, both child-adult and adult-adult transmission play dominant roles in increasing $R_{0}$. (G-I) Fixing adult-adult transmission to be weak $\left(\beta_{22}=0.1\right)$, only child-adult transmission plays a dominant role in increasing $R_{0}$.

choice does not reflect a belief about conditions of the pandemic in any particular population; it is merely intended to demonstrate effects of mitigation strategies within our modelling framework.

\section{Reopening Under Prevalence-Informed Criteria}

Identifying conditions under which schools can be safely reopened is paramount to proposing public health policy for containing the epidemic. In particular, reopening schools only to quickly close down after a few days of instruction is costly both in resources and its negative health effect. Here we investigate the influence of initial 
conditions and elevated child-child transmission on the stopping time $t_{\text {threshold }}$ under an ideal scenario with a $100 \%$ sensitive test. Fig. 3 reports values for $t_{\text {threshold }}$ after varying 222 child-child transmission in active school cohorts by a factor of $c=1, c=2$, and $c=10 . \quad 223$ Reopening schools under high infection burden leads to smaller values for $t_{\text {threshold, }}$ as ${ }^{224}$ expected. Interestingly, these results suggest that multiple cohorts have a desired effect ${ }^{225}$ of delaying school closures beyond the time span of 26 weeks ( 6 months) in our simulations. For example, assuming $0.1 \%$ prevalence at reopening leads to school closure after 6-7 weeks under a single cohort whereas multiple cohorts or the hybrid approach have $t_{\text {threshold }}>26$ weeks. The behavior of the stopping time is insensitive to the contact multiplier $c$. However, there is a sharp transition from $t_{\text {threshold }}>26$ weeks to $t_{\text {threshold }} \approx 4$ weeks under multiple cohorts as prevalence at reopening increases.

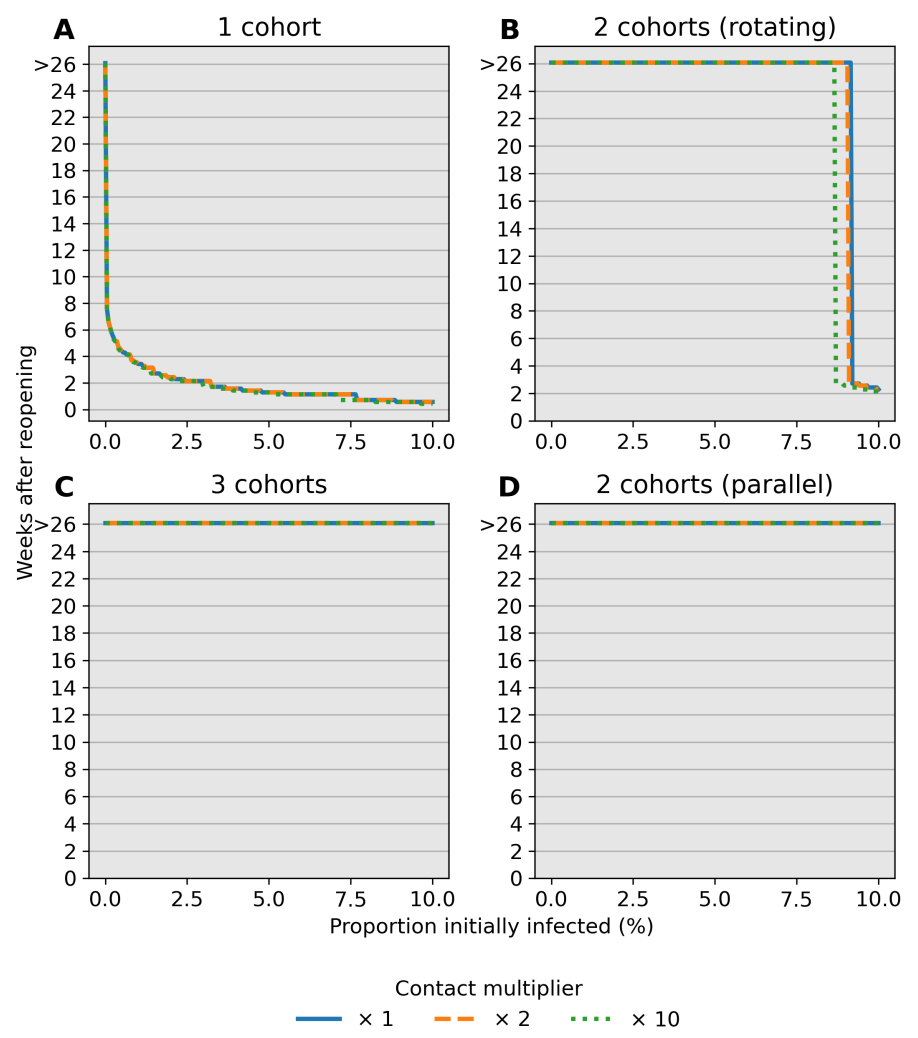

Fig 3. Number of weeks to reach a 5\% stopping threshold in a community. Each scenario assumes a $100 \%$ sensitive test. The stopping time $t_{\text {threshold }}(y$-axis) is simulated under varying prevalence conditions at reopening ( $x$-axis). The contact multiplier for child-child transmission is also varied from (A) $c=1$ to (B) $c=2$ and (C) $c=10$ and has little influence on stopping times. Multiple cohorts are effective at prolonging school operations while staying below a $5 \%$ prevalence threshold over a 14-day window. Note that only detected cases in children contribute to the decision rule. 
Next, we investigate the influence of test sensitivity in our simulated monitoring program and closure criteria on period prevalence, taken as the sum of infecteds and removed individuals. We compare predictions of our model over 26 weeks (6 months) when (1) no action is taken (Fig. 4A-B), (2) the monitoring program employs a perfectly sensitive test without delays in reporting (Fig. 4 C-D), and (3) the monitoring program employs a rapid but less sensitive test (Fig. $4 \mathrm{E}-\mathrm{F})$. Our simulations with a single cohort indicate that a $5 \%$ percent threshold policy can shift period prevalence in children from $55 \%$ to $45 \%$ over the simulated 26 -week period (Fig. $4 \mathrm{~A}-\mathrm{C}$ ). Compared to this ideal scenario, an imperfect test with $50 \%$ detection leads to a slightly later stopping time owing to infections spread by undetected cases and greater overall pediatric infections (Fig. $4 \mathrm{E}$ ). The effect is less pronounced in the adult population due to high adult-adult transmission. Crucially, reopening with a surveillance program may provide approximately 2 weeks of continuous instruction. In our model, infections after closing are driven by a lack of interventions outside of school; testing and isolation in this context can curtail this growth. Our results support the importance of testing and complete school closure in preventing a major disease outbreak after reopening.

We repeat the same simulation study with the hybrid parallel cohort policy. Fig. 5 reports the same indices recorded under the same parameter values as in the single cohort policy. Reducing the force of infection through the community's contact network successfully decreases period prevalence, sustained contact between children notwithstanding (Fig. 5A-D). The stopping rule for the in-person cohort is not triggered even when detection is imperfect (Fig. $5 \mathrm{E}-\mathrm{F})$. Infections are generally higher in the in-person cohort compared to the remote cohort for both children and adults.

\section{Mitigating Transmission Between Children}

Although face masks can reduce the spread of SARS-CoV-2 by $40 \%$ in adults 35, risks of mask wearing by elementary school children include impaired learning, speech development, social development, and facial recognition 36, 37. It is also unclear 


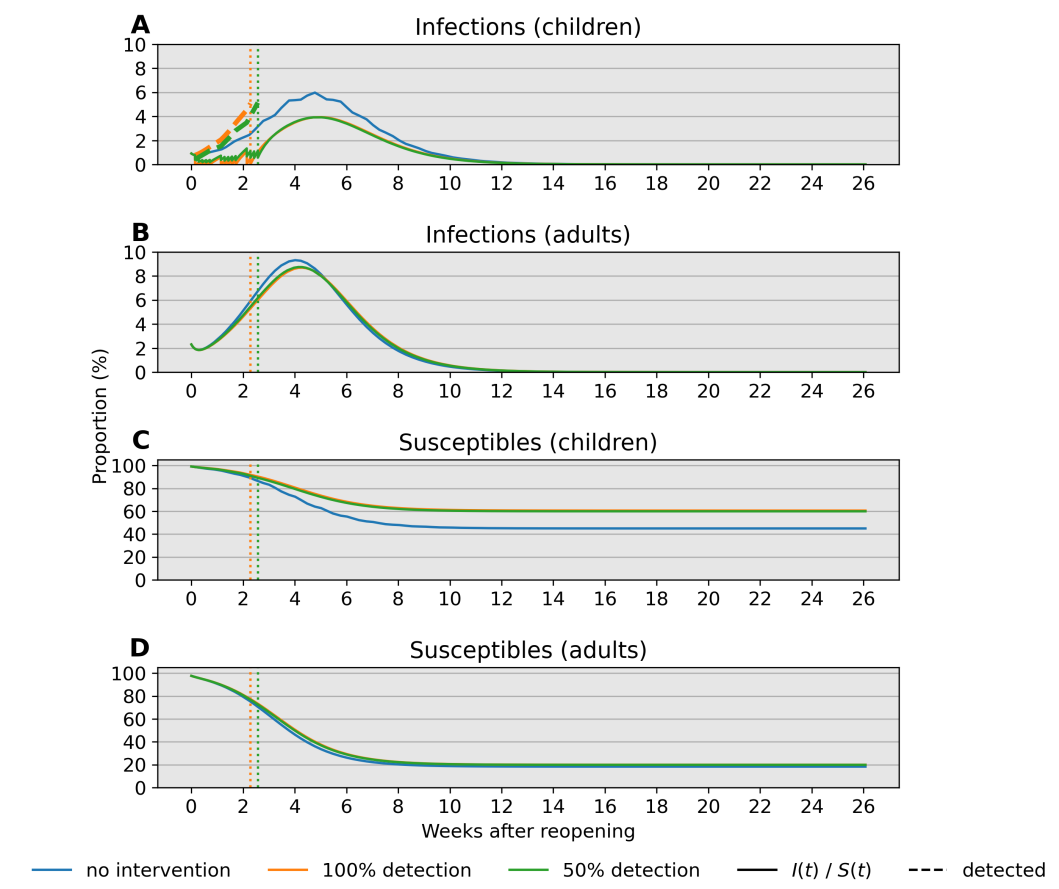

Fig 4. Comparison of infections and susceptibles under different test sensitivities in both children and adults. Simulations are based on parameter values $f_{11}=0.1, f_{12}=0.25, f_{21}=0.15$, and $f_{22}=0.5$ with bulk transmission rate $\beta_{0}=1.2$. Reopening takes place at a $2 \%$ prevalence level (2000 infections per 100,000). The decision criterion over a 14-day sliding window is highlighted in a dotted line. Blue, orange, and green lines correspond to scenarios without intervention, with a $100 \%$ sensitive test, and a 50\% sensitive test, respectively. (A) The 14-day prevalence criteria hits the $5 \%$ threshold after just over 2 weeks in the two testing scenarios. (B) Prevalence in adults peaks after about 4 weeks independent of test sensitivity in children. (C) Testing is effective in keeping most children safe from infection regardless of test sensitivity. (D) Testing in children has little impact on keeping adults free from infection under these conditions.

whether children can consistently wear masks. An October 2020 survey of middle school and high school students, communicated by the CDC, underscores this point with mask wearing varying from approximately $65 \%$ in classrooms and hallways to $25 \%$ in outdoor settings within school boundaries 41 .

We explore the impacts of varying degrees of protection conferred by combined risk reduction strategies, such as mask wearing, desk shields, handwashing, vigilant surface cleaning, improved ventilation, and outdoor instruction. Combined impacts of these strategies are modeled as $20 \%, 40 \%, 60 \%$, and $80 \%$ reductions in the transmission rates $\beta_{11}$ and $\beta_{22}$ relative to reference values. Specifically, we take $\beta_{11}=0.12, \beta_{12}=0.3$, $\beta_{21}=0.18$, and $\beta_{22}=0.6$ as natural rates and apply a $40 \%$ reduction factor to adults 

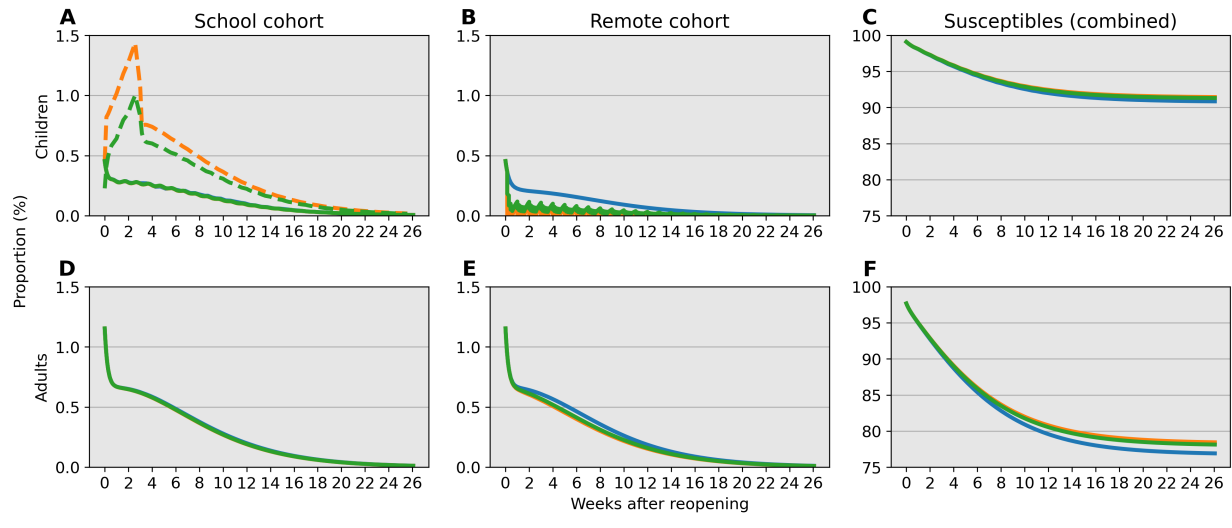

- no intervention

- $100 \%$ detection $\quad-50 \%$ detection

Fig 5. Comparison of cumulative under the parallel cohort approach. (A-C) The 14-day prevalence criteria increases over the first 4 weeks, but point prevalence consistently trends downward due to cohort structure. Over $90 \%$ of children are kept safe from infection under the conditions of this simulation. (D-F) The combination of testing in children and cohort separation prevents a high level of infection in adults.

by setting $\beta_{21}=0.072$ and $\beta_{22}=0.24$. This implies $R_{0} \approx 1.7$ prior to reopening.

Increased contact is modeled by taking $c=10$ so that $\beta_{11}=1.2$, which corresponds to $R_{0} \approx 2.2$ under the full capacity reopening scenario. This represents an extreme that illustrates effects in a poor situation.

Fig. 6 compares prevalence trajectories for interventions directly targeting transmission under a single or two rotating child cohorts. With a single cohort and no mitigation in children, our choices lead to approximately $8 \%, 24 \%$, and $28 \%$ infected children after 4, 13, and 26 weeks following reopening, respectively (Fig. 6A, blue line). However, with measures that lead to an $80 \%$ reduction in transmission, infections at 4 , 13, and 26 weeks are 5\%,11\%, and 13\%, respectively (Fig. 64 A, purple line). Targeting transmission rates in children also reduces infections in adults to a similar degree (Fig. 6B). Much stricter adherence to transmission mitigation measures is required for low infection levels when there is a single cohort (Fig. 6A-B) than when there are two cohorts (Fig. 6C-D). A combination of both types of interventions ultimately results in even fewer infections. 


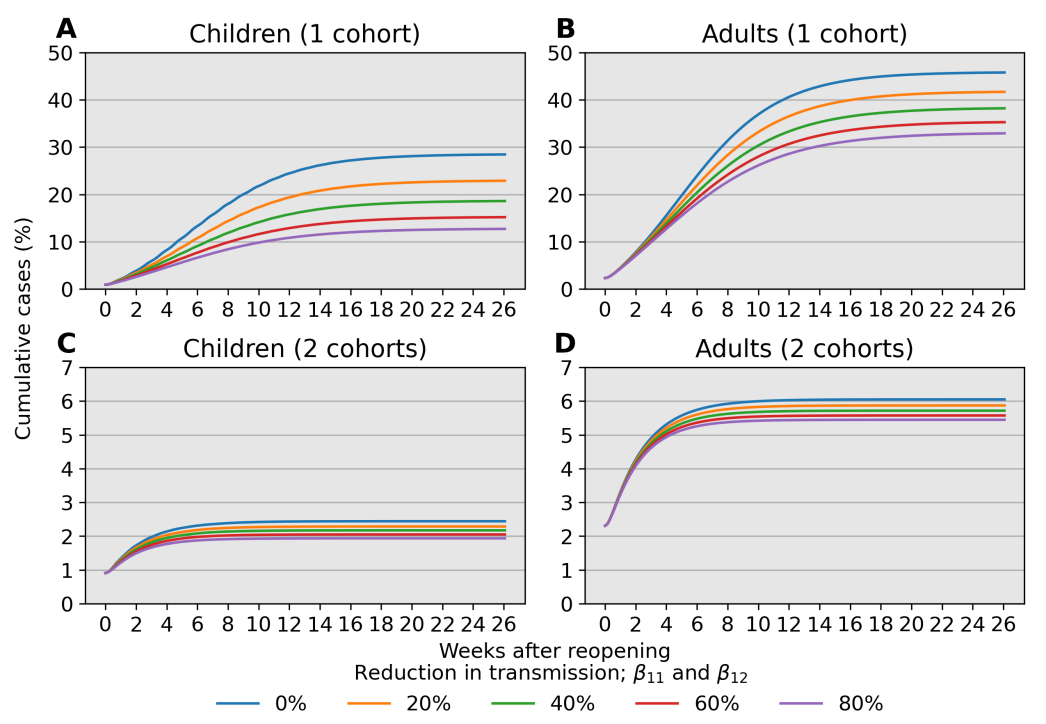

Fig 6. Cumulative prevalence trajectories under risk reduction strategies for children while at school. For child-child transmission, we set $\beta_{11}=0.1 \times(1-r)$ outside of school and $\beta_{11}=(1-r) \times c \times 0.1$ during school, where $r$ is a reduction factor due to effective risk reduction strategies and $c=10$ accounts for increased contact between children. (A-B) Mitigation that reduces transmission between children can lead to a substantial reduction in infections for both children and adults, provided the mitigation effects are large. (C-D) The impact of risk reduction strategies persists when children are separated into 2 rotating cohorts but does not demand as strict an adherence to be effective. An $80 \%$ reduction in pediatric transmission has a weaker effect compared to separating children into 2 rotating cohorts as the latter strategy result in fewer than $5 \%$ pediatric infection over 26 weeks (6 months).

\section{Discussion}

\section{Summary}

Our analysis identifies child-adult transmission as a potential risk to reopening schools even under the plausible assumption of weak child-child transmission relative to adult-adult transmission (Fig 2D-F). Moreover, our simulation studies highlight the profound impact of reducing cohort size with parallel or rotating cohorts under a range of transmission rates and reproduction numbers. For example, during a 6-month time span, reopening schools in a population with $0.1 \%$ infections with 2 cohorts avoids triggering a prevalence closure decision rule based on a $5 \%$ pediatric infection threshold. This, allows schools to stay open longer compared to reopening at $100 \%$ capacity without cohort separation (Fig. 3). Simultaneous adherence to transmission mitigation measures and multiple separated cohorts can keep cases low, for example under $3 \%$ 
(Fig. 6C-D). Our work also underscores the importance of tracking infections and setting a threshold for reverting to remote learning. In the absence of any intervention to in-person instruction, the proportion of school safe from infection stays just above $40 \%$ at equilibrium (Fig. $4 \mathrm{~B}$, blue). This compares with keeping the susceptible proportion above $60 \%$ under the combination of a rapid testing program, a stopping rule, and a single cohort (Fig. $4 \mathrm{~B}$, green and orange).

\section{Limitations}

There are several limitations to our modelling that could be addressed in future studies. $\quad 307$ Finer age stratification is required to predict outcomes in specific communities and can 308 be implemented within our modelling framework. For example, high school students 309 may wear masks and practice physical distancing more reliably than elementary school children, and may also have transmission rates closer to those of adults 43. Second, we assume equal transmission rates among all adults and omit explicit interactions between students and teachers within a classroom, which are critical in implementing backup protocols that allow switches to remote learning. Network-based models are better suited to accounting for classroom and household structures in a population, as well as shifting contact patterns 4648 Third, our model treats school communities in isolation. Schools in urban settings have diverse commuting patterns and face potential for importing cases from outside adjacent neighborhoods. Fourth, our conclusions about reproduction numbers, period prevalence, stopping times, and impact of various mitigation strategies should be understood as offering policy guidance rather than precise quantitative predictions. Our ODEs are suited to fitting prevalence data rather than incidence data which poses a challenge to predictive capabilities. Lastly, our models omit the stochastic nature of infections in small populations. Although these caveats limit the quantitative accuracy of our predictions, we contend that our qualitative conclusions are correct.

\section{Conclusion}

We find that measures reducing class density by rotating cohorts between in-person and remote schooling are likely to have greater impact in reducing the spread of 
SARS-CoV-2 than policies such as mask wearing, handwashing, and physical distancing $\quad{ }_{329}$ in the classroom. Nevertheless, the latter policies combined with a reduction in class $\quad 330$ density are still quite effective in reducing effective transmission. From the perspective of mathematical epidemiology, this is to be expected as separating a contact graph into disconnected pieces ultimately limits the proliferative potential of an infectious disease. Surprisingly, parallel cohorts are as effective as rotating cohorts in case reduction, while requiring less coordination and work schedule adjustment for parents. Educating children under either cohort strategy should be a priority in school re-openings. Benefits of switching to remote learning when infections climb to an unacceptable level benefit from rapid testing, even if imperfect. Our rapid testing predictions are consistent with a recent study 38 on the influence of viral kinetics, test sensitivity, test frequency, and sample-to-answer reporting time in surveillance protocols, which also demonstrates that test efficacy is a secondary concern given the dangers of the pandemic.

Finally, communities should be treated differently. High-risk communities with large class sizes need to be especially careful in exposing children to unnecessary risks. Future work is needed to review policies of schools that have successfully remained open over the past year. Our modeling techniques may be helpful in estimating the expected impact of applying those policies in larger districts.

\section{References}

1. Auger KA, Shah SS, Richardson T, et al. Association between statewide school closure and COVID-19 incidence and mortality in the US. JAMA. 2020 Jul;7. doi:10.1001/jama.2020.14348

2. Kuhfeld M, Tarasawa B. The COVID-19 slide: What summer learning loss can tell us about the potential impact of school closures on student academic achievement. NWEA. 2020.

3. Kuhfeld M, Soland J, Tarasawa B, Johnson A, Ruzek E, Liu J. Projecting the Potential Impact of COVID-19 School Closures on Academic Achievement. Educational Researcher. 2020 Nov 1;49(8):549-65. 
4. Sheikh A, Sheikh A, Sheikh Z, Dhami S. Reopening schools after the COVID-19 lockdown. Journal of Global Health. 2020 Jun;10(1):010376.

5. Office of Governor Gavin Newsom. Governor Gavin Newsom Lays Out Pandemic Plan for Learning and Safe Schools. California Governor. Published July 17, 2020. Accessed July 21, 2020. https://www.gov.ca.gov/2020/07/17/governor-gavinnewsom-lays-out-pandemic-plan-for-learning-and-safe-schools/

6. Seow J, Graham C, Merrick B, Acors S, Pickering S, Steel KJA, et al. Longitudinal observation and decline of neutralizing antibody responses in the three months following SARS-CoV-2 infection in humans. Nature Microbiology. 2020 Dec;5(12):1598-607.

7. Gaebler C, Wang Z, Lorenzi JCC, Muecksch F, Finkin S, Tokuyama M, et al. Evolution of antibody immunity to SARS-CoV-2. Nature. 2021 Jan 18;1-6.

8. Dan JM, Mateus J, Kato Y, Hastie KM, Yu ED, Faliti CE, et al. Immunological memory to SARS-CoV-2 assessed for up to 8 months after infection. Science. 2021 Feb 5;371(6529). Available from: 10.1126/science.abf4063

9. Sokal A, Chappert P, Barba-Spaeth G, Roeser A, Fourati S, Azzaoui I, et al. Maturation and persistence of the anti-SARS-CoV-2 memory B cell response. Cell. 2021 Feb 2;0(0). Available from: https://www.cell.com/cell/abstract/S0092-8674(21)00093-3

10. Davies NG, Klepac P, Liu Y, Prem K, Jit M, Eggo RM. Age-dependent effects in the transmission and control of COVID-19 epidemics. Nature Medicine.

Published online June 16, 2020:1-7. doi:10.1038/s41591-020-0962-9

11. Jones TC, Mühlemann B, Veith T, et al. An analysis of SARS-CoV-2 viral load by patient age. medRxiv. Published online June 9, 2020:2020.06.08.20125484. doi:10.1101/2020.06.08.20125484

12. Zhang J, Litvinova M, Liang Y, et al. Changes in contact patterns shape the dynamics of the COVID-19 outbreak in China. Science. 2020;368(6498):1481-1486. doi:10.1126/science.abb8001 
13. Rackauckas C, Nie Q. DifferentialEquations.jl - A Performant and Feature-Rich Ecosystem for Solving Differential Equations in Julia. Journal of Open Research Software. 2017;5(1):15. doi:10.5334/jors. 151

14. Rackauckas C, Nie Q. Confederated modular differential equation APIs for accelerated algorithm development and benchmarking. Advances in Engineering Software. 2019;132:1-6. doi:10.1016/j.advengsoft.2019.03.009

15. SciML. DifferentialEquations.Jl. Checkout v6.15.0; 2020. https://github.com/SciML/OrdinaryDiffEq.jl

16. SciML. OrdinaryDiffEq.Jl. Checkout v5.41.0; 2020. https://github.com/SciML/DifferentialEquations.jl

17. US Centers for Disease Control and Prevention. Coronavirus Disease 2019 (COVID-19) in the U.S. Centers for Disease Control and Prevention. Published August 3, 2020. Accessed August 4, 2020. https://www.cdc.gov/coronavirus/2019-ncov/cases-updates/cases-in-us.html

18. Kwok KO, Lai F, Wei WI, Wong SYS, Tang JWT. Herd immunity - estimating the level required to halt the COVID-19 epidemics in affected countries. Journal of Infection. 2020;80(6):e32-e33. doi:10.1016/j.jinf.2020.03.027

19. Syal K. COVID-19: Herd immunity and convalescent plasma transfer therapy. Journal of Medical Virology. 2020;92(9):1380-2.

20. Zimmermann P, Curtis N. Coronavirus Infections in Children Including COVID-19: An Overview of the Epidemiology, Clinical Features, Diagnosis, Treatment and Prevention Options in Children. The Pediatric Infectious Disease Journal. 2020;39(5):355-368. doi:10.1097/INF.0000000000002660

21. Verdoni L, Mazza A, Gervasoni A, et al. An outbreak of severe Kawasaki-like disease at the Italian epicentre of the SARS-CoV-2 epidemic: an observational cohort study. The Lancet. 2020;395(10239):1771-1778.

doi:10.1016/S0140-6736(20)31103-X

22. Paltiel AD, Zheng A, Walensky RP. Assessment of SARS-CoV-2 Screening Strategies to Permit the Safe Reopening of College Campuses in the United 
States. JAMA Netw Open. 2020;3(7):e2016818-e2016818.

doi:10.1001/jamanetworkopen.2020.16818

23. Yung CF, Kam K, Nadua KD, et al. Novel Coronavirus 2019 Transmission Risk in Educational Settings. Clin Infect Dis. Published online June 25, 2020. doi:10.1093/cid/ciaa794

24. Zhang J, Litvinova M, Wang W, et al. Evolving epidemiology and transmission dynamics of coronavirus disease 2019 outside Hubei province, China: a descriptive and modelling study. The Lancet Infectious Diseases. 2020;20(7):793-802. doi:10.1016/S1473-3099(20)30230-9

25. Szablewski CM. SARS-CoV-2 Transmission and Infection Among Attendees of an Overnight Camp — Georgia, June 2020. MMWR Morb Mortal Wkly Rep. 2020;69. doi:10.15585/mmwr.mm6931e1

26. Lopez AS. Transmission Dynamics of COVID-19 Outbreaks Associated with Child Care Facilities - Salt Lake City, Utah, April-July 2020. MMWR Morb Mortal Wkly Rep. 2020;69. doi:10.15585/mmwr.mm6937e3

27. Park YJ, Choe YJ, Park O, Park SY, Kim Y-M, Kim J, et al. Contact Tracing during Coronavirus Disease Outbreak, South Korea, 2020 - Volume 26, Number 10-October 2020 - Emerging Infectious Diseases journal - CDC. Available from: $10 /$ gg $5 g b k$

28. Li R, Pei S, Chen B, et al. Substantial undocumented infection facilitates the rapid dissemination of novel coronavirus (SARS-CoV-2). Science.

2020;368(6490):489-493. doi:10.1126/science.abb3221

29. Lauer SA, Grantz KH, Bi Q, et al. The Incubation Period of Coronavirus Disease 2019 (COVID-19) From Publicly Reported Confirmed Cases: Estimation and Application. Annals of Internal Medicine. 2020;172(9):577-582. doi:10.7326/M20-0504

30. Bar-On YM, Flamholz A, Phillips R, Milo R. SARS-CoV-2 (COVID-19) by the numbers. Eisen MB, ed. eLife. 2020;9:e57309. doi:10.7554/eLife.57309 
31. Du Z, Xu X, Wu Y, Wang L, Cowling BJ, Meyers LA. Serial Interval of COVID-19 among Publicly Reported Confirmed Cases. Emerging Infectious Diseases. 2020;26(6):1341-1343. doi:10.3201/eid2606.200357

32. He X, Lau EHY, Wu P, et al. Temporal dynamics in viral shedding and transmissibility of COVID-19. Nature Medicine. 2020;26(5):672-675. doi:10.1038/s41591-020-0869-5

33. Zhu Y, Bloxham CJ, Hulme KD, et al. Children are unlikely to have been the primary source of household SARS-CoV-2 infections. medRxiv. Published online January 1, 2020:2020.03.26.20044826. doi:10.1101/2020.03.26.20044826

34. Karin O, Bar-On YM, Milo T, et al. Adaptive Cyclic Exit Strategies from Lockdown to Suppress COVID-19 and Allow Economic Activity. Epidemiology; 2020. doi:10.1101/2020.04.04.20053579

35. Mitze T, Kosfeld R, Rode J, Wälde K. Face Masks Considerably Reduce COVID-19 Cases in Germany: A Synthetic Control Method Approach. Bonn, Germany: IZA Institute of Labour Econoimics. Published online 2020.

36. American Academy of Pediatrics. COVID-19 Planning Considerations: Guidance for School Re-entry. Published June 25, 2020. Accessed July 1, 2020. http://services.aap.org/en/pages/2019-novel-coronavirus-covid-19infections/clinical-guidance/covid-19-planning-considerations-return-to-inperson-education-in-schools/

37. American Federation of Teachers. Pediatricians, Educators and Superintendents Urge a Safe Return to School This Fall. American Federation of Teachers. Published July 10, 2020. Accessed July 13, 2020. https://www.aft.org/pressrelease/pediatricians-educators-and-superintendents-urge-safe-return-school-fall

38. Larremore DB, Wilder B, Lester E, et al. Test sensitivity is secondary to frequency and turnaround time for COVID-19 surveillance. medRxiv. Published online January 1, 2020:2020.06.22.20136309. doi:10.1101/2020.06.22.20136309

39. HAN Archive - 00432 - Health Alert Network (HAN). https://emergency.cdc.gov/han/2020/han00432.asp (2020). February 2, 2021. 
40. Dufort, E. M. et al. Multisystem Inflammatory Syndrome in Children in New York State. New England Journal of Medicine (2020) 10.1056/nejmoa2021756.

41. COVID-19 Stats: Percentage of Middle and High School Students Aged 13-21 Years Attending In-Person Classes Who Reported Observing Fellow Students Wearing a Mask All the Time, by School Setting and Activity — United States, October 2020. MMWR Morb Mortal Wkly Rep. 2021;70. Available from: 10.15585/mmwr.mm7006a5

42. Ludvigsson JF. Systematic review of COVID-19 in children shows milder cases and a better prognosis than adults. Acta Paediatrica. 2020;109(6):1088-95.

43. Viner RM, Mytton OT, Bonell C, Melendez-Torres GJ, Ward J, Hudson L, et al. Susceptibility to SARS-CoV-2 Infection Among Children and Adolescents Compared With Adults: A Systematic Review and Meta-analysis. JAMA Pediatr. 2021 Feb 1;175(2):143.

44. Children and COVID-19: State-Level Data Report. American Academy of Pediatrics. February 4, 2021.

45. van den Driessche P. Reproduction numbers of infectious disease models. Infectious Disease Modelling. 2017 Aug 1;2(3):288-303.

46. Bu F, Aiello AE, Xu J, Volfovsky A. Likelihood-Based Inference for Partially Observed Epidemics on Dynamic Networks. Journal of the American Statistical Association. 2020 Aug 18;1-17.

47. Britton T. Epidemic models on social networks - With inference. Statistica Neerlandica. 2020;74(3):222-41.

48. Jiang Y, Kassem R, York G, Junge M, Durrett R. SIR epidemics on evolving graphs. arXiv:190106568. 2019 Jan 19; Available from:

http://arxiv.org/abs/1901.06568

49. Lee B, Hanley JP, Nowak S, Bates JHT, Hébert-Dufresne L. Modeling the impact of school reopening on SARS-CoV-2 transmission using contact structure data from Shanghai. BMC Public Health. 2020 Nov 16;20(1):1713. 
medRxiv preprint doi: https://doi.org/10.1101/2020.08.05.20169086; this version posted March 19, 2021. The copyright holder for this preprint (which was not certified by peer review) is the author/funder, who has granted medRxiv a license to display the preprint in perpetuity.

It is made available under a CC-BY 4.0 International license.

50. U.S. Department of Education, National Center for Education Statistics. Digest of Education Statistics, 2018. 2019 Dec 24; Available from:

https://nces.ed.gov/pubsearch/pubsinfo.asp?pubid=2020009 